\title{
Finger necrosis after accidental radial artery puncture
}

elSSN: 2383-4625

Received: 29 October 2014

Revised: 3 December 2014

Accepted: 3 December 2014

Correspondence to: Yeon Kwon Jeong Department of Emergency Medicine, Samsung Medical Center,

Sungkyunkwan University School of Medicine, 81 Irwon-ro, Gangnam-gu, Seoul 135-710, Korea

E-mail: hi.jeong@samsung.com The second finger was eventually amputated owing to gangrene. Radial artery puncture can occur accidentally during IV wrist access, resulting in severe morbidity. Providers should carefully examine the puncture site and collateral flow, followed by multiple examinations to ensure distal circulation.

Keywords Arterial occlusive diseases; Catheterization; Fingers; Thromboembolism

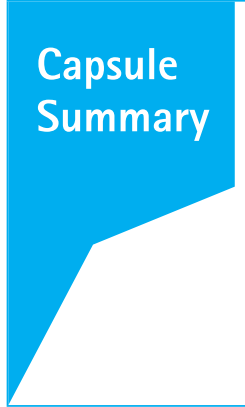

What is already known

The incidence of complications after radial artery cannulation and risk factors for complication has been studied before.

What is new in the current study

Critical complications can be encountered if appropriate precaution as the Modified Allen's Test or Doppler ultrasonography is not accompanied.

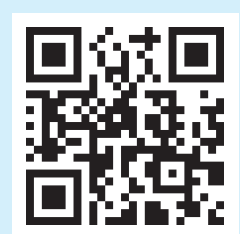

How to cite this article:

Kang JS, Lee TR, Cha WC, Shin TG, Sim MS, Jo IJ, Song KJ, Rhee JE, Jeong YK. Finger necrosis after accidental radial artery puncture. Clin Exp Emerg Med 2014;1(2):130-133.

This is an Open Access article distributed under the terms of the Creative Commons Attribution Non-Commercial License (http:// creativecommons.org/licenses/by-nc/3.0/). 


\section{INTRODUCTION}

Radial artery puncture is an invasive procedure that is frequently used for arterial blood gas analysis or invasive blood pressure monitoring. Although known to be safe, radial artery cannulation can cause thrombosis, leading to severe ischemia of the hand and even subsequent gangrene and tissue loss. The incidence of radial artery cannulation complications is reportedly $0.1 \%-20 \% 0^{1,2}$ The purposes of this case report are to share the treatment course of finger necrosis after accidental radial artery puncture and review the literature.

\section{CASE REPORT}

A previously healthy 63-year-old woman presented to the emergency department (ED) complaining of aggravating pain on her left second and third fingers along with a color change. She had visited a local clinic for a diagnostic esophagoduodenoscopy the day before as part of her annual checkup that was accompanied by procedural sedation. After having trouble establishing intravenous (IV) access for medication, the providers managed to insert an IV catheter in the left wrist on the lateral palmar side, a less

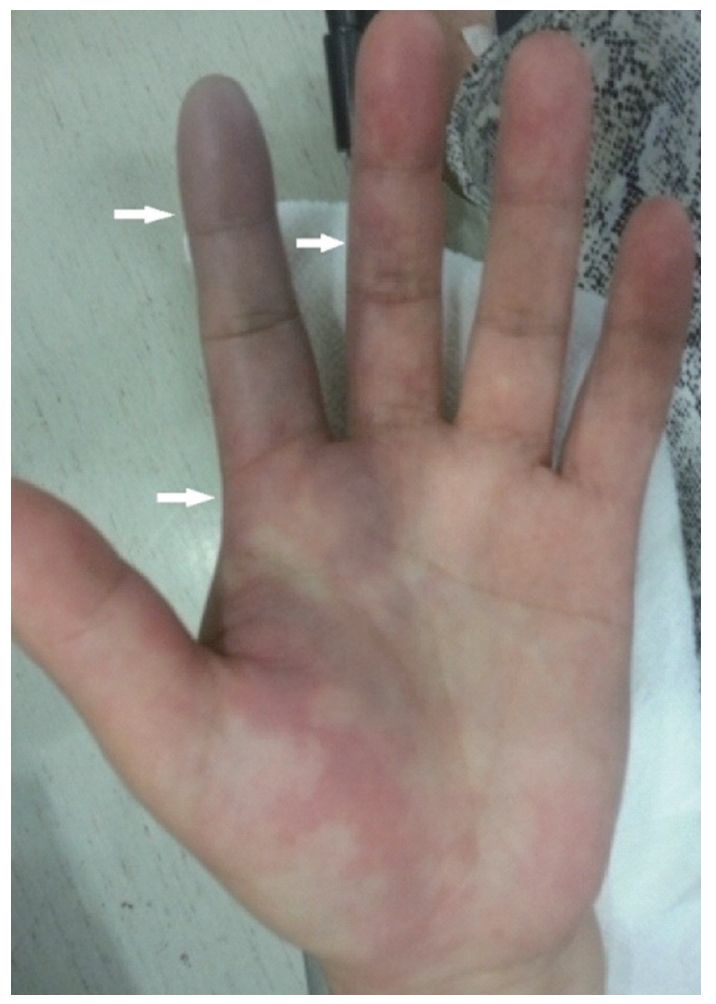

Fig. 1. Discoloration of the second and third fingers evident at the time of presentation to the emergency department (hospital day \#1). The margin of the affected site is clearly visible (arrows). common site for IV access. During the procedure, she experienced a sharp and extreme pain that persisted even after removal of IV access. However, the treating physician did not verify the distal circulation of her hand and discharged her after prescribing a painkiller.

After returning home, her fingers turned pale and eventually started to show a bluish discoloration (Fig. 1). The pain became intolerable; therefore, she visited our ED, 12 hours after the procedure. Her initial vital signs were as follows: blood pressure, 127/77 $\mathrm{mmHg}$; heart rate, 101/min; respiratory rate, 16/min; and body temperature, $36.7^{\circ} \mathrm{C}$. Although seemed stable, she complained of excessive pain (8/10 on the numeric rating scale) in her left second and third fingers. A physical examination revealed significant sensory deterioration and coldness on the affected sites. Puncture marks were visible near her radial artery. Emergency Doppler ultrasonography performed in the ED showed normal blood flow from her radial artery to the common palmar digital artery. Decreased flow at the second and third proper palmar digital arteries was also found. Computed tomography angiography showed a pseudoaneurysm in her left radial artery and no sign of blood flow from her palmar digital artery (Fig. 2). A thromboembolism resulting in digital necrosis was suspected. Subcutaneous enoxaparin $(40 \mathrm{mg})$ and oral nifedipine $(30 \mathrm{mg})$ were administered. The occluded branch was distal, which limited the usefulness of surgery; therefore, angiography was performed instead.

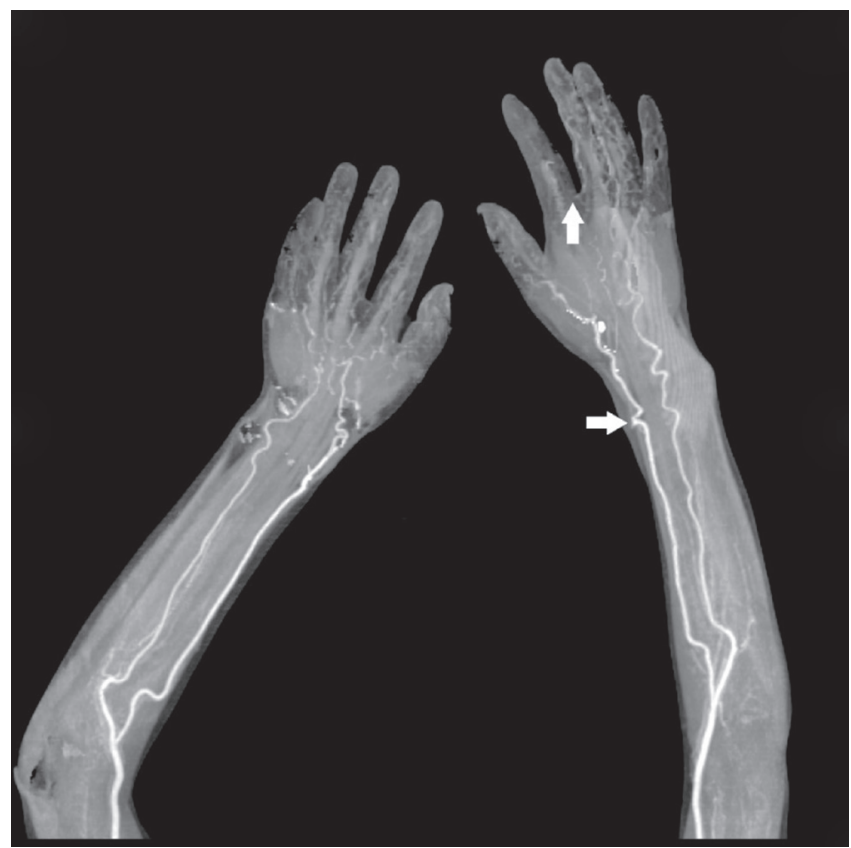

Fig. 2. A computed tomography angiography image taken 1 hour after presentation to the emergency department shows absence of blood flow within the affected sites (arrows). 
Angiography showed patent radial and ulnar arteries, with a patent common palmar digital artery and sufficient collateral circulation. However, the second and third proper palmar digital arteries were not visualized at all. Vasodilation was induced with nitroglycerin $(300 \mu \mathrm{g})$ but failed. A small pseudoaneurysm observed in the radial artery near the puncture site suggested that the second and third finger occlusion was caused by a microembolism from the pseudoaneurysm. Intra-arterial thrombolysis was performed with the infusion of urokinase $(960,000 \mathrm{U})$ over 4 hours. However, follow-up angiography did not show any improvement (Fig. 3).

The patient was admitted for close observation. Aspirin (100 mg per day [QD]), clopidogrel (75 mg QD), and alprostadil (10 $\mu \mathrm{g}$ QD) were prescribed. After close observation for 1 week, the circulation on the third finger almost completely recovered, but the second finger turned gangrenous, which forced us to amputate it (Fig. 4). The patient was discharged 12 days after admission in a

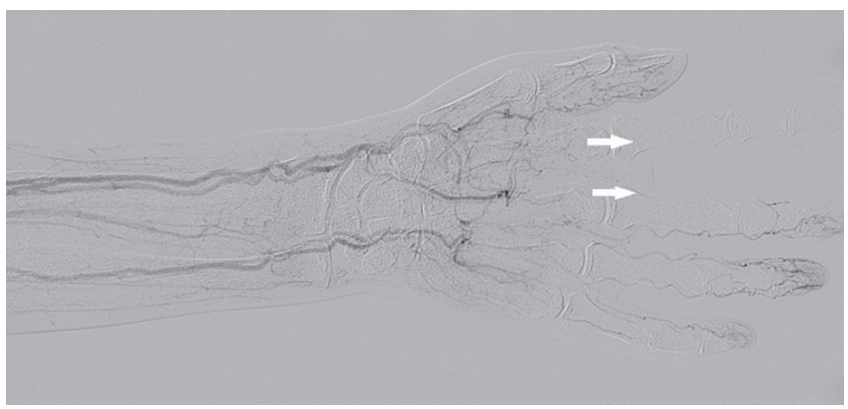

Fig. 3. Angiography image of the affected sites. Blood flow to the second and third fingers was negligible after the injection of thrombolytic agents 9 hours after she presented to the emergency department (arrows).

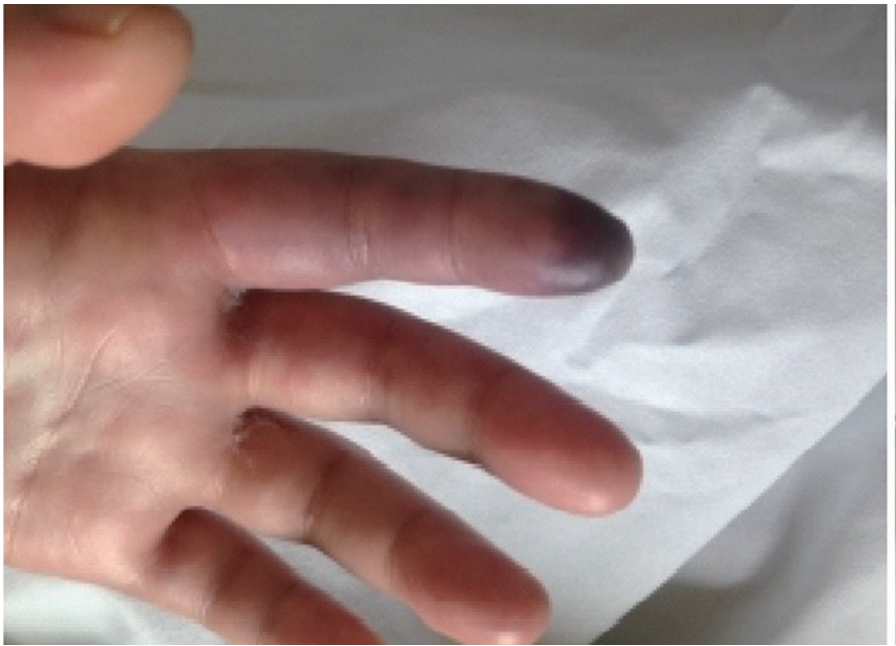

tolerable state and was scheduled for outpatient surgery. The middle and distal phalanges of her second finger were amputated 25 days after the event, and she was discharged without additional significant complications.

\section{DISCUSSION}

Radial artery puncture is frequently performed in many EDs. Arterial blood gas analysis and invasive blood pressure monitoring require radial artery puncture. The radial artery is favored because of the existence of the collateral and ulnar arteries as well as the ease of access. The radial artery is also a popular access site for coronary angiography and cerebral angiography. Doppler ultrasonography or a modified Allen's test can be used to evaluate the patency of collateral flow. However, the efficacy of the Allen's test remains under debate. ${ }^{3,4}$

Common complications after radial artery cannulation include temporary radial artery occlusion (19.7\%), hematoma (14.4\%), infection $(0.72 \%)$, hemorrhage $(0.53 \%)$, and bacteremia $(0.13 \%)$. The complications described herein-permanent ischemic damage and pseudoaneurysm-are very rare adverse effects, with rates of $0.09 \%$ each. ${ }^{1}$ Risk factors for complications involve the cannula size, vasospasm, patient age, female sex, high number of puncture attempts, and duration of catheter placement. . $^{5-8}$

In this case, the patient's providers attempted IV access for procedural sedation. Probably because of poor vein access, they chose an unfamiliar site for the IV, which resulted in critical pseudoaneurysm and thromboembolism. Multiple attempts as well as female sex could have contributed to the outcome.

The optimal treatment of patients who develop ischemia after radial artery cannulation is controversial; therefore, it should be

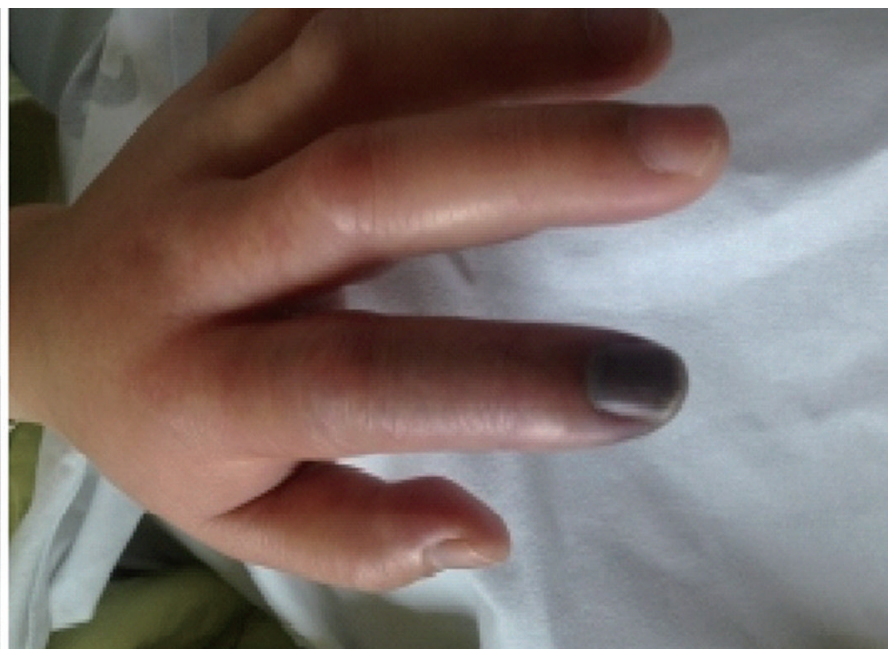

Fig. 4. Gangrenous change in the second finger, and recovery in the third finger. 
individualized. ${ }^{9}$ Systemic anticoagulation, intra-arterial verapamil, and balloon angioplasty with localized abciximab or low molecular weight dextran are the first-line therapies for patients without bleeding risks. However, the effect of these therapies is limited when angiographic intervention is unsuccessful. ${ }^{10,11}$ Invasive angiography can visualize the injured vessels directly and provide a vehicle for the direct injection of vasodilatory agents to the site. The limitation is that it cannot reach very distal portions as in this case, which attenuates its efficacy. When a substantially large portion of tissue is ischemic, surgical treatments as thromboembolectomy, arterial repair, patch angioplasty, or vein grafts can be considered. ${ }^{12}$

In conclusion, radial artery puncture must be conducted in proper sites and accompanied by collateral flow check-up, carefully conducted procedures, follow-up, and continuous monitoring of distal blood flow. When a complication is suspected, rapid assessment and optimal treatments are required to prevent fatal complications.

\section{CONFLICT OF INTEREST}

No potential conflict of interest relevant to this article was reported.

\section{REFERENCES}

1. Scheer B, Perel A, Pfeiffer UJ. Clinical review: complications and risk factors of peripheral arterial catheters used for haemodynamic monitoring in anaesthesia and intensive care medicine. Crit Care 2002;6:199-204.

2. Brown $A E$, Sweeney $D B$, Lumley J. Percutaneous radial artery cannulation. Anaesthesia 1969;24:532-6.

3. Bedford RF, Wollman H. Complications of percutaneous radial-artery cannulation: an objective prospective study in man. Anesthesiology 1973;38:228-36.

4. Slogoff $S$, Keats $A S$, Arlund $C$. On the safety of radial artery cannulation. Anesthesiology 1983;59:42-7.

5. Dahl MR, Smead WL, McSweeney TD. Radial artery cannulation: a comparison of 15.2- and 4.45-cm catheters. J Clin Monit 1992;8:193-7.

6. Li AH, Yeih DF, Chen KC, Chu SH, Weng CS. The thicker the skin fold, the less hematoma development: a novel parameter other than body mass index associated with transradial vascular complication. Angiology 2007;58:597-602.

7. Bedford RF. Radial arterial function following percutaneous cannulation with 18- and 20-gauge catheters. Anesthesiology 1977;47:37-9.

8. Tuncez A, Kaya Z, Aras D, et al. Incidence and predictors of radial artery occlusion associated transradial catheterization. Int J Med Sci 2013;10:1715-9.

9. Valentine RJ, Modrall JG, Clagett GP. Hand ischemia after radial artery cannulation. J Am Coll Surg 2005;201:18-22.

10. Gallacher BP. Intra-arterial verapamil to reverse acute ischaemia of the hand after radial artery cannulation. Can J Anaesth 1991;38:138.

11. Jaradat Z, Basir B, Revtyak G. Treatment of radial artery occlusions using balloon angioplasty and localized intra-arterial abciximab. J Interv Cardiol 2014;27:217-22.

12. Lee MK, Lee IO, Kong MH, Han SK, Lim SH. Surgical treatment of digital ischemia occurred after radial artery catheterization. J Korean Med Sci 2001;16:375-7. 